\title{
ADOECIMENTO DOCENTE DE UMA INSTITUIÇÃO PÚBLICA FEDERAL DE ENSINO SUPERIOR: UMA ANÁLISE DOS ATESTADOS MÉDICOS DE SAÚDE
}

\author{
Elen Villegas Campos ${ }^{1}$ \\ Alexandra Maria Almeida Carvalho ${ }^{2}$ \\ Albert Schiaveto de Souza ${ }^{3}$
}

RESUMO: O professor é um dos principais agentes de mudança social sendo fundamental no processo de ensino e aprendizagem. Esse profissional facilita o desenvolvimento intelectual, emocional, social, cultural, político e profissional de alunos. Conhecer o processo de adoecimento docente assim como formular estratégias de melhoria na saúde destes profissionais se torna relevante para o desenvolvimento social. Objetivo: Verificar o adoecimento docente através da análise de atestados médicos. Método: Estudo transversal que analisou atestados de saúde de professores da Universidade Federal de Mato Grosso do Sul, entre os anos de 2007 e 2017. Resultados: Registrou-se 2525 atestados, representando um total de 62956 dias de trabalho perdidos. Os docentes adoecem principalmente por doenças mentais $19,8 \%$ (499), sendo o transtorno depressivo recorrente e os episódios depressivos os mais frequentes. A segunda causa de adoecimento foram doenças do sistema osteomuscuları,5\% (264) sendo mais frequentes os problemas relacionados à coluna vertebral. Conclusão: Doenças mentais e comportamentais foram as mais prevalentes entre os docentes.

Palavras-chaves: Doença. Docentes. Universidade.

\footnotetext{
${ }^{1}$ Doutoranda em Saúde e Desenvolvimento na Região Centro-Oeste, na Universidade Federal de Mato Grosso do Sul (UFMS). Mestre em Saúde e Desenvolvimento na Região Centro-Oeste pela UFMS. Especialista em Enfermagem do Trabalho pela Universidade Católica Dom Bosco (UCDB). Especialista em Gestão do Trabalho e da Educação em Saúde pela Universidade Federal do Rio Grande do Norte (UFRN). Graduada em Enfermagem pela UFMS. Professora do curso de Medicina da Universidade Estadual de Mato Grosso do Sul (UEMS).E-mail: elenvillegas@hotmail.com.

2 Doutora em Biosystems Eng. and Environmental Science, The University of Tennessee. Mestre em Engenharia Elétrica pela Universidade Federal de Uberlândia (UFU). Graduada em Engenharia Civil pela Universidade Estadual Paulista Júlio de Mesquita Filho (UNESP). Professora adjunta da Universidade Federal de Mato Grosso do Sul (UFMS) e coordenadora do Programa de Pós- Graduação Saúde e Desenvolvimento na Região Centro-Oeste. Atualmente desenvolve projetos em saúde ambiental, saúde do trabalhador e avaliação de serviços de saúde.

3 Possui graduação em Fisioterapia pela Universidade Estadual de Londrina (1988), mestrado (200I) e doutorado (2004) em Ciências (Fisiologia) pela Universidade de São Paulo, campus Ribeirão Preto. Em 2013 concluiu um ano de pós-doutorado na University of Oxford, UK, por meio da Universidade de São Paulo, Campus Ribeirão Preto. Trabalhou no Hospital Adventista do Pênfigo de 1989 a 1998 (Campo Grande, MS). Atuou como docente na Universidade Católica Dom Bosco (UCDB) de 1996 a 2008 (Campo Grande, MS). Desde 2008 é professor da Universidade Federal de Mato Grosso do Sul (UFMS), atualmente como professor associado, ao nível de graduação, orientador credenciado no Programa de Pós-graduação em Saúde e Desenvolvimento na Região Centro-Oeste da UFMS. É o atual pró reitor de assuntos estudantis da UFMS. É avaliador do INEP/MEC para o curso de Fisioterapia. Tem experiência nas áreas de Fisioterapia e Neurofisiologia, atuando principalmente nos seguintes temas: plasticidade neuronal, memória espacial, fisiologia dos núcleos da base do cérebro e bioestatística.
} 


\section{INTRODUÇÃO}

O Brasil é o maior país da América do Sul e o quinto do mundo em extensão territorial, estendendo- se por uma área de $8.514 .876,599 \mathrm{~km}^{2}$. São mais de 206 milhões de habitantes segundo o último Censo de 20ı. A população é formada pela interação entre os povos europeus, africanos e nativos indígenas, tendo recebido várias correntes imigratórias (alemães, italianos, espanhóis, japoneses e sírio-libaneses) que contribuíram também para a formação étnica atual da população. A maioria dos brasileiros é negra (50,74\%). Os brancos correspondem a $47,73 \%$ dos habitantes. O Brasil é uma República Federativa Presidencialista, cujo idioma é o português (PNDU, 2018).

A Universidade surge no Brasil como resultado da formação das elites que foram formadas em instituições europeias. A primeira universidade brasileira, Universidade do Rio de Janeiro, foi fundada em 1920, no Rio de Janeiro, sendo focada somente no ensino e na orientação profissional (STALLIVIERI, 2007).

Ao longo de sua história não houve grandes mudanças na educação, sendo essa, marcada por uma prática elitista e excludente. A educação no Brasil é instituída pela primeira vez como direito social na Constituição Federal de 1934, sendo revigorada mais tarde pela Carta Magna de 1988 que definiria a universalização do ensino para todas as camadas que compõem a sociedade brasileira (FERREIRA JR., 2010).

A educação no Brasil é regida pela Lei de Diretrizes e Bases da Educação Nacional (LDB), Lei no 9.394/1996, que define como dever do Estado a garantia da educação básica obrigatória e gratuita dos 4 (quatro) aos 17 (dezessete) anos de idade. A educação está organizada da seguinte forma: pré-escola; ensino fundamental; médio e superior que abrange cursos de graduação e pós-graduação (LDB, 2017).

Segundo o Censo da Educação Superior 2017, no Brasil existem 2.448 Instituições de Ensino Superior, com 199 universidades (públicas e privadas).

O docente com sua capacidade intelectual, técnica e profissional para desenvolver projetos e pesquisas, se torna um patrimônio do Sistema Educacional, dando aos cidadãos a oportunidade de educar-se (NUNES et al., 2014).

O professor, diferente de um operário de indústria, trabalha com sujeitos e não objetos. Ele tem como objetivo transformar alunos, educá-los e instruí-los, sendo o trabalho docente caracterizado por relações humanas, individuais e sociais ao mesmo tempo, os alunos são seres heterogêneos, socialmente, culturalmente, etnicamente e economicamente. Assim também possuem diferenças cognitivas, o que caracteriza um trabalho de grande investimento afetivo e emocional por parte dos professores com os alunos (TARDIF, 2007).

Fortalecendo esta ideia, Antunes (2010) descreve o trabalho como sendo atividade vital, exclusivamente humana, que transforma o mundo e o sujeito.

Grande parte da vida de uma pessoa é dedicada ao trabalho, sendo esse caracterizado na maioria das vezes por longas jornadas, incluindo períodos noturnos, ritmo intenso e altos níveis de exigência, provocando sofrimento, mal-estar e adoecimento (BRITO FILHO; MACIEL; FELIZARDO, 20I7). 
Atualmente, o desempenho de uma atividade profissional dispensa pouco tempo para o lazer e o convívio familiar, pois a maior parte do dia é passada a trabalhar. Esta realidade faz com que o trabalho ocupe um lugar de destaque no dia a dia de quem o desenvolve (POCINHO; FRAGOEIRO, 2012).

Não muito diferente de outras atividades profissionais, o trabalho docente é caracterizado por uma sobrecarga relacionada à produção científica exigida pelas instituições reguladoras da pós-graduação e de fomento à pesquisa, requisito para professores se manterem em programas de pós-graduação, favorecendo benefícios de financiamentos para pesquisas (FONTANA; PINHEIRO, 20I0).

A sobrecarga também fica evidente quando Vilela (2010) cita as atividades dos docentes nas universidades públicas, como a ministração de aulas, correção de provas e trabalhos, realização de pesquisas, escrita de artigos, participação em eventos da universidade, orientação de alunos na produção de trabalhos científicos, participação de projetos de extensão e buscar a atualização de novos conhecimentos, tudo na tentativa de acompanhar as mudanças da globalização.

O trabalho do professor do ensino superior sé configurado por Oliveira e Freitas (2008) como sendo muito difícil e de grande responsabilidade, uma vez que tem como responsabilidade formar profissionais para um setor produtivo da sociedade. Além disso, muitos alunos que adentram as universidades carecem de capacidade de reflexão e de abstração; domínio da língua portuguesa e de línguas estrangeiras; bagagem sociocultural; hábitos de estudo; respeito ao outro; compromisso; e disciplina dificultando o trabalho docente.

A elevada carga de trabalho provoca mal-estar na profissão docente, resultado das exigências impostas pelo atual contexto social. Os principais elementos que têm causado esse mal-estar são o acelerado avanço do saber, que faz com que o professor busque aperfeiçoamento contínuo (muitas vezes a qualificação exigida é buscada com pouco ou sem nenhum apoio institucional); a constatação de que a realidade se transformou e a impossibilidade de continuar mantendo objetivos de ensino e aprendizagem que não correspondem mais ao contexto social; as exigências sociais pelo sucesso profissional dos alunos num contexto de acirramento da competitividade no mundo do trabalho (ESTEVE, 1987).

Beraldo (2009) associa o trabalho docente ao produtivismo moderno, caracterizado, na educação, pela racionalização de recursos; desvalorização profissional; intensificação do trabalho, transformação da ciência em mercadoria; criação da cultura do individualismo, da competitividade, da inclusão excludente; controle da produtividade com base em indicadores meramente quantitativos; incentivo à venda de serviços; tendência em atribuir ao professor a culpa pelas mazelas e pelos fracassos da educação.

Essas mudanças resultaram em um mal-estar docente, caracterizado por: sentimentos de desajustamento, insatisfação, abandono da profissão, absenteísmo, esgotamento, estresse, ansiedade e depressão (NÓVOA et al., 1995).

Um ranking de valorização elaborado pela fundação educacional Varkey Gems em 2013, com base em quatro indicadores-interesse pela profissão, respeito em sala de aula, remuneração salarial e comparação com outras profissões, o Brasil ficou em penúltimo lugar, ficando à frente apenas de Israel (NEHER, 2014). 
No Brasil, segundo o CENSO 2017, existem 214.608 docentes (ativos e afastados). A carreira docente é exercida por profissionais de diversas formações profissionais, das mais variadas áreas.

Silvério et al. (2010) relatam que os professores consideram que trabalhar com pessoas prejudica a saúde, principalmente quando as interações contrariam seus princípios e disponibilidades, o que resulta em "baixa imunidade" e repercussões nas suas condições de saúde geral, favorecendo diversas doenças, incluindo a depressão.

Fontana e Pinheiro (2010) destacaram como sofrimento psíquico predominante a ansiedade e a depressão, estes foram justificados pela sobrecarga de trabalho. Nos finais de semana, fora das horas normais de trabalho, os professores preparam aulas, deveres de casa, documentação, material pedagógico, provas e correção de trabalho (TARDIF; LESSARD, 2007).

O trabalho docente é uma atividade que propicia ao mesmo tempo prazer e sofrimento. Os professores vivenciam o sofrimento devido às exigências do trabalho e prazer advindo das compensações à subjetividade, proporcionado pelas trocas de ideias, pela ampliação do conhecimento e pela oxigenação do pensamento (VILELA, 2010).

Vilela (2010) cita que as atividades docentes são marcadas pela lógica da avaliação quantitativa, vinculada ao pagamento de gratificações e ao financiamento de pesquisas conforme a produtividade, reforçando, assim, o individualismo e a competição entre os pares, que vem se naturalizando no cotidiano do trabalho na universidade.

Trabalhos sobre as condições de trabalho e saúde de docentes são escassos comparados com outras áreas trabalhistas. Porém conhecer a saúde dos mesmos é relevante, visto que o professor se tornou um trabalhador submetido às mesmas condições dos trabalhadores fabris, pois a escola adquire a nova função de formar trabalhadores. $\mathrm{O}$ aluno passa a ser visto como produto e a escola como uma instituição produtora da força de trabalho (LIMA, M. F. E. M. e LIMA, F. D. O., 2009).

\section{MATERIAL E MÉTODOS}

Estudo observacional, transversal, que teve como objetivo analisar o perfil de afastamento do trabalho por motivo de doença de docentes da Universidade Federal de Mato Grosso do Sul. Foram analisados os atestados de saúde entre os anos de 2007 até 2017, obtidos do sistema de registros de atestados médicos da Pró- Reitoria de Gestão de Pessoas (PROGEP) de todos os campi da universidade.

A Universidade Federal de Mato Grosso do Sul (UFMS) teve sua origem em 1962 e está sediada na Cidade de Campo Grande, Mato Grosso do Sul, possuindo sedes em Campo Grande, Aquidauana, Chapadão do Sul, Corumbá (do Pantanal), Coxim, Naviraí, Nova Andradina, Paranaíba, Ponta Porã e Três Lagoas. Em 2018, o corpo docente era composto de mil setecentos e quarenta e três (1743) professores. Sendo Io43 somente em Campo grande e 700 nas demais localidades.

Na UFMS, a PROGEP é responsável por manter os registros de atestados médicos funcionais dos servidores em banco de dados informatizado. Os dados dos afastamentos 
foram disponibilizados em planilha Excel, obtida do sistema de afastamento conforme autorização junto ao pró- reitor de gestão de pessoas e comitê de ética.

Os critérios de inclusão foram todos os atestados que caracterizaram motivo de afastamento por doença com CID especificado, na Universidade Federal de Mato grosso do Sul, no período de 2007 a 2017. Já os critérios de exclusão foram os atestado fora do período analisado.

As variáveis analisadas nesse estudo foram: idade; Sexo; regime de trabalho (dedicação exclusiva, 40 horas, 20 horas); motivo de afastamento (CID ro - Décima Revisão da Classificação Internacional de Doenças e de Problemas Relacionados a Saúde); tempo de afastamento(dias) e taxa de dias de afastamento por atestado.

A análise da associação entre as variáveis categóricas foi realizada por meio do teste qui- quadrado. Análise estatística foi realizada no programa estatístico SPSS, versão 23.0, considerando um nível de significância de $5 \%$.

O projeto foi aprovado pelo comitê de ética da UFMS situado na cidade de Campo Grande, Mato Grosso do Sul, Brasil, sob número CAE 85633518.8.oooo.oo21.

\section{RESULTADOS}

No período de janeiro de 2007 a dezembro de 2017, foram contabilizados 2525 atestados de saúde de professores, totalizaram 62.956 dias de ausência no trabalho.

Houve mais atestados do sexo feminino $(64,1 \%-n=1618)$ do que do sexo masculino $(35,9 \%-\mathrm{n}=907$ - teste binomial, $\mathrm{p}<\mathrm{o}, 00 \mathrm{I})$. A maior parte dos atestados foi de docentes com idade acima de 40 anos $(82,6 \%$ - $\mathrm{n}=2086)$, com idade média de $51,80 \pm 11,00$ anos (média \pm desvio padrão da média).

Em relação à carga horária de trabalho, a maioria dos atestados era de docentes em regime de dedicação exclusiva $(87,4 \%-\mathrm{n}=2208)$. Quanto à titulação, a maior parte dos atestados era de docentes doutores $(66,3 \%-n=1673)$.

Houve associação significativa entre o sexo dos docentes e a faixa etária dos mesmos (teste qui-quadrado, $\mathrm{p}<\mathrm{o}, \mathrm{ooI}$ ), sendo que na faixa etária de $4 \mathrm{I}$ a 50 anos foram observados mais atestados em docentes do sexo feminino $(31,7 \%$ - $n=513)$ do que em docentes do sexo masculino $(23,8 \%-\mathrm{n}=216)$. Por outro lado, na faixa etária acima de 6o anos foram observados mais atestados em docentes do sexo masculino $(31,3 \%-n=284)$ do que em docentes do sexo feminino $(23,3 \%-n=377$ - teste do qui-quadrado com correção de Bonferroni, $\mathrm{p}<0,05)$. Estes resultados estão apresentados na Tabela I.

No ano de 2018, independentemente do tipo de vínculo, carga horária e titulação, houve 1743 docentes vinculados à instituição em estudo, destes 49,3\% (n=859) eram mulheres e 50,7\% $(n=884)$ eram homens. Diante disso, era esperado que a proporção de atestados por sexo fosse equivalente a esta distribuição, ou seja, cerca de 50\% dos atestados para cada sexo. No entanto, neste estudo, foram observados significativamente mais atestados em docentes do sexo feminino $(64,1 \%$ - $n=1618)$ do que em docentes do sexo masculino (35,9\% - $\mathrm{n}=907$ - teste binomial, $\mathrm{p}<\mathrm{o}, \mathrm{oor})$.

Houve associação entre o sexo dos docentes e o período de afastamento dos atestados (teste do qui-quadrado, $\mathrm{p}=0,002$ ), sendo que houve mais atestados de até 5 dias em docentes do sexo feminino $(33,1 \%-n=536)$ do que em docentes do sexo masculino 
$(29,2 \%-n=265)$. Por outro lado, houve mais atestados de mais de 15 dias em docentes do sexo masculino $(46,5 \%-n=422)$ do que em docentes do sexo feminino $(38,9 \%-n=630-$ teste do qui-quadrado, com correção de Bonferroni, $\mathrm{p}<0,05)$. Estes resultados estão apresentados na Tabela I.

Tabelar: Porcentagem e número de atestados por sexo segundo faixa etária e número de dias de atestados de docentes, Mato Grosso do Sul, 2007-2017- $(n=2525)$.

\begin{tabular}{|c|c|c|c|c|}
\hline \multirow{2}{*}{ Variáveis } & \multicolumn{2}{|c|}{ Sexo } & \multirow{2}{*}{ Total } & \multirow{2}{*}{ Valor de $\mathrm{p}$} \\
\hline & Feminino & Masculino & & \\
\hline \multicolumn{5}{|l|}{ Faixa etária } \\
\hline De 20 a 30 anos & $\mathrm{I}, 4(22)^{\mathrm{a}}$ & $2, \mathrm{I}(19)^{\mathrm{a}}$ & $\mathrm{I}, 6(4 \mathrm{I})$ & \multirow{5}{*}{$<0$, oOI } \\
\hline De 3r a 40 anos & $16,3(263)^{a}$ & $\mathrm{I} 4,8(\mathrm{I} 34)^{\mathrm{a}}$ & $15,7(397)$ & \\
\hline De 4I a 50 anos & $31,7(513)^{a}$ & $23,8(2,16)^{b}$ & $28,9(729)$ & \\
\hline De 51 a 60 anos & $27,4(443)^{a}$ & $28,0(254)^{\mathrm{a}}$ & $27,6(697)$ & \\
\hline Acima de 60 anos & $23,3(377)^{b}$ & $3 \mathrm{I}, 3(284)^{\mathrm{a}}$ & 26,I (66o) & \\
\hline \multicolumn{5}{|c|}{ Período de afastamento } \\
\hline Até 5 dias & $33, \mathrm{I}(536)^{\mathrm{a}}$ & $29,2(265)^{b}$ & $3 \mathrm{I}, 7$ (8oI) & \multirow{4}{*}{0,002} \\
\hline De 6 a io dias & $\mathrm{I2,2}(\mathrm{I} 97)^{\mathrm{a}}$ & $9,4(85)^{a}$ & $\mathrm{II}, 2(282)$ & \\
\hline De II a I5 dias & $15,8(255)^{a}$ & $14,9(135)^{a}$ & $15,4(390)$ & \\
\hline Mais de 15 dias & $38,9(630)^{b}$ & $46,5(422)^{\mathrm{a}}$ & $4 \mathrm{I}, 7(\log 2)$ & \\
\hline Total & I0o,o (1618) & I00,o (907) & $100,0(2525)$ & \\
\hline
\end{tabular}

Os resultados estão apresentados em frequência relativa (frequência absoluta). Valor de p no teste do quiquadrado. Letras diferentes na linha indicam diferença entre os sexos (teste do qui-quadrado, com correção de Bonferroni, $\mathrm{p}<0,05)$.

Do total de 2525 atestados analisados neste estudo, os mesmos estavam distribuídos em 2i capítulos do CID-ı, com percentuais variando entre $19,8 \%(n=499)$ e $0,1 \%(n=3)$, sendo os capítulos mais prevalentes, o Capítulo V - Transtornos mentais e comportamentais (Foo-F99) (19,8\% - n=499), o Capítulo XIII - Doenças do sistema osteomuscular e do tecido conjuntivo (Moo-M99) (Io,5\% $\mathrm{n}=264$ ) e o Capítulo II Neoplasias [tumores] (Coo-D48) $(7,1 \%-n=179)$. Estes resultados juntamente com os dias 
de afastamento e a média de dias de afastamento por atestado estão apresentados na Tabela 2.

Tabela 2: Distribuição do número de atestado e dias de afastamento segundo os capítulos do CID-Io, Mato Grosso do Sul, 2007-2017- $(\mathrm{n}=2525)$.

$\begin{array}{cccc}\text { Capítulo CID ro } & \begin{array}{c}\text { Atestados } \\ \%(n)\end{array} & \begin{array}{c}\text { Dias } \\ \%(n)\end{array} & \begin{array}{c}\text { Dias por } \\ \text { atestado }\end{array}\end{array}$

Capítulo V - Transtornos mentais e 19,8 (499) $\quad 31,4(19752) \quad 39,59$ comportamentais (Foo-F99)

Capítulo XIII - Doenças do sistema osteomuscular e do tecido conjuntivo (Moo-M99)

Capítulo II - Neoplasias [tumores] (Coo$\left.\mathrm{D}_{4} 8\right)$

Os resultados estão apresentados em frequência relativa (frequência absoluta).

$\begin{array}{ccc}\text { 10,5 (264) } & \text { 10,4 (6572) } & 24,89 \\ 7,1 \text { (179) } & \text { 14,5(9145) } & 51,09\end{array}$

Dentre os 499 atestados do Capítulo V (Transtornos mentais e comportamentais), os CIDs mais frequentes foram o F33- Transtorno depressivo recorrente $(27,5 \%-n=137)$, o $\mathrm{F}_{32}$ - Episódios depressivos $(25,7 \%$ - $\mathrm{n}=128)$, o $\mathrm{F}_{43}$ - Reação ao stress grave e transtorno de adaptação ( $13,2 \%-n=66)$, o $F_{41-}$ - Outros transtornos ansiosos (II,8\% - n=59) e oF 31 Transtorno afetivo bipolar $(9,4 \%-\mathrm{n}=47)$.

Docentes do sexo feminino apresentaram um percentual maior de atestados dos CIDs F33- Transtorno depressivo recorrente (35,3\% - n=98) e F43- Reações ao "stress" grave e transtornos de adaptação $(16,65-\mathrm{n}=46)$, do que docentes do sexo masculino (CID F33: 17,7\% - $n=39$; CID F43: 9,1\% - $n=20$; teste do qui-quadrado, $p<0,001$ e $p=0,020$, respectivamente). Por outro lado, docentes do sexo masculino apresentaram um percentual maior de atestados dos CIDs $\mathrm{F}_{32}$ - Episódios depressivos $(31,2 \%-\mathrm{n}=69)$ do que docentes do sexo feminino ( $21,2 \%-n=59$; teste do qui-quadrado, $\mathrm{p}=0,015)$. Estes resultados estão apresentados na Tabela 3 .

Tabela 3-Porcentagem e número de atestados por sexo segundo os cinco subgrupos mais prevalentes do Capítulo V. Mato Grosso do Sul, 2007-2017 ( $\mathrm{n}=499)$.

\begin{tabular}{|c|c|c|c|}
\hline \multirow[b]{2}{*}{ CID-ro } & \multicolumn{2}{|c|}{ Sexo } & \multirow[b]{2}{*}{ Valor de $\mathrm{p}$} \\
\hline & $\begin{array}{c}\text { Feminino } \\
(\mathrm{n}=278)\end{array}$ & $\begin{array}{c}\text { Masculino } \\
(\mathrm{n}=22 \mathrm{I})\end{array}$ & \\
\hline $\mathrm{F}_{33}$ - Transtorno depressivo recorrente & $35,3(98)^{\mathrm{a}}$ & $17,7(39)^{b}$ & $<0$, ooI \\
\hline $\mathrm{F}_{32}-$ Episódios depressivos & $21,2(59)^{b}$ & $31,2(69)^{a}$ & 0,015 \\
\hline $\begin{array}{l}\text { F } 43 \text { - Reações ao "stress" grave e } \\
\text { transtornos de adaptaçãoo }\end{array}$ & $\mathrm{I} 6,6(46)^{\mathrm{a}}$ & $9, \mathrm{I}(20)^{\mathrm{b}}$ & 0,020 \\
\hline $\mathrm{F}_{4 \mathrm{I}}-$ Outros transtornos ansiosos & $13,0(36)^{a}$ & $\mathrm{IO}, 4(23)^{\mathrm{a}}$ & 0,463 \\
\hline
\end{tabular}




\begin{tabular}{llll}
\hline $\mathrm{F}_{31}$ - Transtorno afetivo bipolar & $9,0(25)^{\mathrm{a}}$ & I0,0 $(22)^{\mathrm{a}}$ & 0,833 \\
\hline
\end{tabular}

Os resultados estão apresentados em frequêcia relativa (frequêcia absoluta). Valor de $p$ no teste do quiquadrado. Letras diferentes na linha indicam diferença entre os sexos (teste do qui-quadrado, $\mathrm{p}<0,05$ ).

O Capítulo XIII- Doenças do sistema osteomuscular e do tecido conjuntivo (Moo a M99) foi o segundo capítulo com mais atestados, sendo 264 do total de 2525 atestados, sendo os CIDs mais frequentes os relacionados à coluna lombar, torácica e cervical (M54-Dorsagia, M51- Outros transtornos de discos intervertebrais, M41- Escoliose, M40- Cifose e lordose, M45-Espondilite anquilosante, M47- Espondilose, M53- Outras dorsopatias não classificadas em outra parte, M48- Outras espondilopatias, M50Transtornos dos discos cervicais) (39,8\% - $n=105)$; articulações e tendões $(24,6 \%-n=65)$ (Mo5- Artrite reumatoide soropositiva, Mo6- Outras artrites reumatoides, Mio- Gota, Mi2- Outras artropatias especificadas, Mi3- Outras artrites, Mi6- Coxartrose (artrose do quadril), M20- Deformidades adquiridas dos dedos das mãos e dos pés, M21- Outras deformidades adquiridas dos membros, $\mathrm{M}_{25}$ - Outros transtornos articulares não classificados em outra parte, M65- Sinovite e tenossinovite, M71-Outras bursopatias, M76Entesopatias dos membros inferiores, excluindo pé e M77- Outras entesopatias); Joelho (12,9\% - n=34) (Mi7- Gonartrose (artrose do joelho), M22- Transtornos da rótula (patela), M23- Transtornos internos do joelho);e Ombro(II,4\% - n=30) (M75-Lesões do Ombro)

Docentes do sexo masculino apresentaram um percentual maior de atestados dos CIDs relacionados à coluna lombar, torácica e cervical $(45,3 \%$ - n $=43)$, Joelho $(22,1-$ $\mathrm{n}=2 \mathrm{I}$ ) e Ombro (I0,5\% - $\mathrm{n}=10$ ), do que docentes do sexo feminino (Coluna lombar, torácica e cervical: $36,7 \%-n=62$; Joelho: $7,7 \%-n=13$; Ombro: $11,8 \%-n=20$; teste do qui-quadrado, $\mathrm{p}=0,216 ; \mathrm{p}=0$,oore $\mathrm{p}=0,905$, respectivamente). Por outro lado, docentes do sexo feminino apresentaram um percentual maior de atestados dos CIDs relacionado às articulações e tendões $(30,2 \%$ - $n=51)$ do que docentes do sexo masculino ( $14,7 \%$ - $n=14$; teste do quiquadrado, $\mathrm{p}=0,08 \mathrm{I})$. Estes resultados estão apresentados na Tabela 4.

Tabela 4- Porcentagem e número de atestados por sexo segundo os cinco subgrupos mais prevalentes do Capítulo XIII. Mato Grosso do Sul, 2007-2017 $(\mathrm{n}=264)$.

\begin{tabular}{|c|c|c|c|}
\hline \multirow[b]{2}{*}{ Local relacionado ao CIDıo } & \multicolumn{2}{|c|}{ Sexo } & \multirow[b]{2}{*}{ Valor de $\mathrm{p}$} \\
\hline & $\begin{array}{c}\text { Feminino } \\
(n=169)\end{array}$ & $\begin{array}{c}\text { Masculino } \\
(\mathrm{n}=95)\end{array}$ & \\
\hline Coluna lombar, torácica e cervical & $36,7(62)^{\mathrm{a}}$ & $45,3(43)^{\mathrm{a}}$ & 0,216 \\
\hline Articulações e tendões & $30,2(5 \mathrm{I})^{\mathrm{a}}$ & $14,7(14)^{b}$ & $0,08 \mathrm{I}$ \\
\hline Joelho & $7,7(13)^{b}$ & $22, \mathrm{I}(2 \mathrm{I})^{\mathrm{a}}$ & o,oor \\
\hline Ombro & $\mathrm{II}, 8(2 \mathrm{O})^{\mathrm{a}}$ & $10,5(10)^{a}$ & 0,905 \\
\hline
\end{tabular}

Os resultados estão apresentados em frequêcia relativa (frequêcia absoluta). Valor de $p$ no teste do quiquadrado. Letras diferentes na linha indicam diferença entre os sexos (teste do qui-quadrado, $\mathrm{p}<0,05$ ).

O terceiro capítulo que apresentou maior número de atestados foi o Capítulo II - Neoplasias [tumores] $($ Coo-D 48$)$ com $7,1 \%(n=179)$. Os CIDs mais frequente foi o 
C50- Neoplasia maligna da mama (34,0\% - $\mathrm{n}=6 \mathrm{I})$, seguido dos CIDs relacionados as neoplasia de útero ( $\mathrm{C}_{5}$-Neoplasiamaligna do corpo do útero, $\mathrm{D}_{25}$ - Leiomioma do útero, D26-Outras neoplasias benignas do útero) $(15,1 \%-n=27)$, neoplasias do aparelho digestivo CIDs (Cio-Neoplasia maligna da orofaringe, Ci5-Neoplasia maligna do esôfago, Ci6Neoplasia maligna do estômago,Ci8-Neoplasia maligna do cólon, Cig-Neoplasia maligna da junção retossigmóide, $\mathrm{C}_{20}-$ Neoplasia maligna do reto, $\mathrm{C}_{25}$-Neoplasia maligna do pâncreas) $(14,5 \%$ - $n=26)$ e neoplasias de pele ( $\mathrm{C}_{44}$ - Outras neoplasias malignas da pele, Do4-Carcinoma in situ da pele, D22- Nevos melanocíticos, D23- Outras neoplasias benignas da pele e $\mathrm{C}_{43}$-Melanoma maligno da pele) $(9,5 \%$ - $n=17)$ e nos homens especificamente a neoplasia de próstata (C6I- Neoplasia maligna da próstata) $(6,1 \%-n=I I)$.

Docentes do sexo masculino apresentaram um percentual maior de atestados dos CIDs relacionados a neoplasias do Sistema digestivo (3I,7\% - $\mathrm{n}=\mathrm{II})$ do que docentes do sexo feminino $(5,2 \%-\mathrm{n}=6$; teste do qui-quadrado, $\mathrm{p}<\mathrm{o}, \mathrm{ooI})$. Estes resultados estão apresentados na Tabela 5 .

Tabela 5 - Porcentagem e número de atestados por sexo segundo os cinco subgrupos mais prevalentes do Capítulo II- Neoplasias [tumores], Mato Grosso do Sul, 2007-2017.

\begin{tabular}{|c|c|c|c|}
\hline \multirow[b]{2}{*}{ Neoplasias } & \multicolumn{2}{|c|}{ Sexo } & \multirow[b]{2}{*}{ Valor de $\mathrm{p}$} \\
\hline & $\begin{array}{c}\text { Feminino } \\
(\mathrm{n}=\mathrm{II} 6)\end{array}$ & $\begin{array}{c}\text { Masculino } \\
(\mathrm{n}=63)\end{array}$ & \\
\hline Maligna da mama & $52,6(61)$ & $0,0(0)$ & - \\
\hline Útero & $23,3(27)$ & $\mathrm{o}, \mathrm{o}(\mathrm{o})$ & - \\
\hline Aparelho digestivo & $5,2(6)^{b}$ & $3 \mathrm{I}, 7(20)^{\mathrm{a}}$ & $<0, \mathrm{OOI}$ \\
\hline Pele & $10,3(12)^{a}$ & $8,0(5)^{a}$ & 0,796 \\
\hline Próstata & $\mathrm{o}, \mathrm{o}(\mathrm{o})$ & I7,5 (II) & - \\
\hline
\end{tabular}

Os resultados estão apresentados em frequêcia relativa (frequêcia absoluta). Valor de $p$ no teste do quiquadrado. Letras diferentes na linha indicam diferença entre os sexos (teste do qui-quadrado, $\mathrm{p}<0,05$ ).

\section{DISCUSSÃO}

Este estudo apresentou a prevalência de atestados pelo sexo feminino (64,1\% $\mathrm{n}=6418)$ entre as idades de $4 \mathrm{I}$ a 50 anos $(31,3 \%$ - $\mathrm{n}=513)$. Viana, Barreto e Nunes (2013) estudaram docentes e técnicos administrativos da educação médicos em uma universidade federal do recôncavo baiano, e também encontrou prevalência do sexo feminino nos atestados $(62,93 \%$ - $n=4789)$, no entanto a faixa etária prevalente foi entre 3r e 40 anos de idade.

Apesar de não podermos afirmar que mulheres adoecem mais que homens, podemos afirmar que os atestados foram em maior número de as mulheres, essa limitação do estudo propõe uma melhor investigação desse número considerando que segundo Silva e Borges (2017) apesar do direito igualitário entre homens e mulheres de ingressar na universidade mediante concurso público, ocorrem diferenças significativas na vida das mulheres como a responsabilidade pelos afazeres domésticos e cuidados a filhos e familiares, gerando sobrecarga e desigualdade. 
De acordo com estudo do IPEA (2017), 94\% das mulheres com renda até um salário-mínimo dedicavam- se a afazeres domésticos, e dentre as com renda superior a oito salários mínimos esse percentual é de 79,5\%.

Do total de 2525 atestados analisados, o Capítulo V (Transtornos mentais e comportamentais) teve maior número de atestados $(19,8 \% \mathrm{n}=499)$ sendo descritos nos CIDs: a depressão, a ansiedade, o estresse e o transtorno afetivo bipolar com mais frequência. Em estudo semelhante Silva (2015) ao analisar livros de junta médica de uma universidade federal, onde havia os registros dos atestados médicos dos profissionais, também encontrou a prevalência de adoecimento de cunho depressivo, afetivo e de humor, entre os docentes, sendo prevalente o CID F32- episódios depressivos.

Diehl e Marin (2016) descrevem alguns fatores determinantes para o adoecimento mental dos professores, entre eles se destacam a forma de organização do trabalho, a falta de reconhecimento, problemas motivacionais e comportamentais dos alunos (falta de limite e de educação, dificuldades de relacionamento), e problemas no ambiente físico.

A National Union of Teachers - NUT (2017) cita a como principais fontes dos altos níveis de estresse docente a sobrecarga de trabalho, o comportamento inadequado por parte de alguns alunos, pressões de metas de avaliação, assédio moral, estresse de avaliação e remuneração relacionada ao desempenho e falta de oportunidades profissionais.

Dos transtornos mentais e comportamentais os CIDs mais prevalentes foram o $\mathrm{F}_{33}$ Transtorno depressivo recorrente $(27,5 \%-\mathrm{n}=137)$ e o $\mathrm{F}_{32}$ - Episódios depressivos (25,7\% $\mathrm{n}=\mathrm{I28}$ ). O manual do Ministério da saúde (200I) relacionou o CID F32 ao trabalho, na profissão docente, havendo um nexo de causa, que são as exigências excessivas de desempenho cada vez maior, geradas pelo excesso de competição.

Silva e Carvalho (2016) em uma revisão de literatura evidenciou a prevalência de depressão entre professores universitários e citou a elevada carga de trabalho, política educacional de desvalorização do trabalho docente, insatisfação com as condições de sala de aula, a falta de equipamentos e recursos pedagógicos, competitividade no mercado de trabalho, as cobranças de publicação como fatores que podem predispor os docentes à depressão.

O segundo capítulo de doenças com maior número de atestados foi o capítulo XIII (Doenças do sistema osteomuscular e do tecido conjuntivo) (Io,5\% $n=264$ ), os CIDs relacionados à coluna vertebral foram os de maior ocorrência (39,8\% - $n=105)$. Esta categoria de CIDs também foi prevalente em servidores da Universidade Federal do Recôncavo da Bahia, sendo a dorsalgia (CID-ıo M54) o mais relatado (VIANA; BARRETO; NUNES, 2013).

Estudo de Fontana e Pinheiro (20I0) sobre condições de saúde auto-referidas de professores de uma universidade comunitária destacou a lombalgia como dor mais referida $(26 \%)$, seguida de cefaleia (19,3\%), artralgias ( $16,1 \%)$, cervicalgia $(16,1 \%)$ e dor nos membros inferiores $(13 \%)$.

Oliveira et al. (2017) associaram as afecções osteomusculares como dores nas costas ao excesso de horas de trabalho em pé ou sentado e condições ambientais em geral, no que tange às condições de trabalho.

Santos et al. (2009) O fato de passarem muito tempo entre a postura em pé ou sentada e, neste caso, utilizando cadeiras e mesas desconfortáveis e que não preenchem 
requisitos indicados ergonomicamente; executarem movimentos repetitivos ao apagar o quadro, o que ainda os faz elevar os braços acima do nível do ombro, devem ser considerados, pois, podem fadigar grupos musculares e mesmo lesionar articulações. Esses aspectos fazem parte do contexto do ambiente ocupacional docente que afetam, em especial, o sistema osteomuscular.

O Capítulo II- Neoplasias foi o terceiro com maior número de atestados $(\mathrm{n}=179)$ de 2525 atestados. Nas mulheres prevaleceram os CIDs de neoplasia de mama ( $52,6 \% \mathrm{n}=6 \mathrm{I})$ e de útero $(23,3 \% \mathrm{n}=27)$, e nos homens a neoplasia de próstata $(\mathrm{I} 7,5 \%-\mathrm{n}=\mathrm{II})$, no entanto esses resultados podem estar relacionados às taxas de incidência estimadas, para o ano de 2018, pelo Instituto Nacional do Câncer (INCA), no estado de Mato Grosso do Sul (6o,9 casos/ Ioo mil habitantes- câncer de mama), (20,49 casos/ Ioo mil habitantes- câncer de útero) e (86,47casos/ Ioo mil habitantes- Câncer de próstata).

\section{CONCLUSÃO}

A análise de 2525 atestados médicos da Universidade Federal de Mato Grosso do Sul no período de 2007 a 2017, revelou a prevalência das doenças mentais e comportamentais dos docentes dessa instituição $(n=499)$ sendo os principais CIDs o F33Transtorno depressivo recorrente, F32- Episódios depressivos, F43- "Reações ao "stress" grave e transtornos de adaptação", F41- Outros transtornos ansiosos e F31- Transtorno afetivo bipolar.

Outra categoria prevalente de Doenças foram as osteomusculares $(n=264)$, sendo a dorsalgia (CID M54) e outras categorias de CIDs relacionadas à coluna vertebral as mais prevalentes $(39,8 \%-\mathrm{n}=105)$.

O fator sobrecarga de trabalho, a grande demanda das universidades, as exigências de produtividade e a competitividade, associados a outros problemas institucionais estão diretamente relacionados ao desgaste mental dos professores.

Já as condições de trabalho e a forma como é executado o trabalho desses profissionais estão ligados aos problemas osteomusculares.

Os professores são um meio importante de promover o desenvolvimento social através da formação profissional e até moral de pessoas. Portanto é relevante que novos estudos verifiquem o processo de adoecimento desses profissionais, fornecendo conhecimento suficiente para estratégias de prevenção e intervenção na saúde dos mesmos.

\section{REFERÊNCIAS}

Antunes R. Adeus ao trabalho?. Ensaio sobre as metamorfoses e a centralidade do mundo do trabalho. za ed. São Paulo: Cortez. p. 120. 2010.

Beraldo TML. Formação de docentes que atuam na Educação Superior, In: Rev de Educ Pública, Cuiabá, v. I8, n. 36, p. 71-88. 2009. 
Brito filho FH, Maciel RHMO, Felizardo JM. Carga de trabalho do professor de educação superior: o processo de construção de um instrumento de medida. Rev Lab Fort, Ceará, V. oI, n. 17, p. II8-142. 2017.

BRASIL. Ministério da Educação. Censo da Educação Superior - Notas Estatísticas. Diretoria de Estatísticas Educacionais (DEED). Disponível em: $\langle$ http://download.inep.gov.br/educacao_superior/censo_superior/documentos/2018/censo _da_e ducacao_superior_2017-notas_estatisticas2.pdf >. Acesso em: dez. 2018.

Diehl L, Marin AH. Adoecimento mental em professores brasileiros: revisão sistemática da literatura. Est Interd Psic. Londrina, v. 7, n. 2, p. 64-85. 2006.

Fausto B. História do Brasil. São Paulo: Editora da Universidade de São Paulo EDUSP. P. 2002.

Ferreira JM. História da Educação Brasileira: da Colônia ao século XX / Amarilio Ferreira Jr. São Carlos: EdUFSCar. p. I23. 2010.

Esteve JM. O mal-estar docente: a sala de aula e a saúde dos professores. Tradução de Durley de Carvalho Cavicchia. Bauru, São Paulo: EDUSC, Brasil. P.30. 1987.

Fontana RT, Pinheiro DA. Condições de saúde auto-referidas de Professores de uma universidade regional. Rev Gaucha de Enf. Porto Alegre (RS) 31(2):270-6. 2010.

INCA. Estimativas para o ano de 2018 das taxas brutas e ajustadas de incidência por Ioo mil habitantes e do número de casos novos de câncer, segundo sexo e localização primária. Campo Grande- MS. 2oro. Disponível em: < http://www.inca.gov.br/estimativa/2018/mato-grosso-sul-campo-grande.asp >. Acesso em: 20 ago. 2018.

IPEA. Retrato das Desigualdades de Gênero e Raça - 1995 a 2015. 2017. Disponível em: <chromextension://oemmndcbldboiebfnladdacbdfmadadm/http://www.ipea.gov.br/port al/images/stories/PDFs/170306_retrato_das_desigualdades_de_ genero_raca.pdf > Acesso em: jun. 2017.

Lima MFEM, Lima-filho DO. Condições de trabalho e saúde do/a professor/a universitário/a. Ciênc \& Cog, I4(3), 62-82. 2009.

BRASIL. Lei de diretrizes e bases da educação nacional. Brasília: Senado Federal, Coordenação de Edições Técnicas. 58 p. 2017.

BRASIL. CID Io. Disponível em: <http://datasus.saude.gov.br/sistemas-eaplicativos/cadastros-nacionais/cid-Io>. Acesso em: 15 set. 2018. 
Neher C. A dura realidade de ser professor no Brasil. 2014. Disponível em: $\langle$ https://p.dw.com/p/IAs7r $>$ Acesso em or de Janeiro de 2018.

Nunes MFO, Hutz CS, Pires JG, Oliveira CM. Subjective Well- Beingand Time Use of Brazilian PhD Professors. Rev Paid (Ribeirão Preto), 24(59), 379-387. 2014.

Nóvoa A et al. Profissão professor. 2 ed. Coleção ciências da educação. Porto editora. Portugal, p.12. 1995 .

NUT. Teacher stress - nut guidance for divisions, associations and reps. Disponível em: 〈https://www.teachers.org.uk/search/node/stress〉. Acesso em: io set. 2018.

Oliveira MAM, Freitas MVT. Políticas contemporâneas para o ensino superior: precarização do trabalho docente?. Rev Ext-Class. n.I, v. 2. P.135-136. 2008.

Pocinho M, Fragoeiro JG. Satisfação dos Docentes do Ensino Superior. Act Colomb de Psic, I5(I), 87-97. 2012.

Rosário M JÁ, Melo CN. 2015. A educação jesuítica no Brasil colônia. Rev HISTEDBR, Campinas, no 61, p. 379-389, ISSN: 1676-2584.

Stallivieri L. O sistema de ensino superior do brasil características, Tendências e perspectivas. D.R. Unión de Universidades de América Latina Y El Caribe. pp.79-roo. 2007.

UFMS. Edital PROGEP/UFMS nº II2, de 26 de dezembro de 2018. Abertura de concurso público para ingresso na carreira do magistério superior da UFMS. SEI/UFMS - 0924123 Edital. 2018.

Santos GLV et al. Ocorrência de distúrbios osteomusculares relacionados ao trabalho dos professores de uma instituição de ensino superior de Belém/PA. Fisiot Bras - V. ıo, n. o4. 2009 .

BRASIL. Programa das Nações Unidas para o Desenvolvimento (PNDU). Disponível em: <http://www.br.undp.org/content/brazil/pt/home/countryinfo.html: Acesso em Setembro de 2018 .

SILVA EP. Adoecimento e sofrimento de professores universitários: dimensões afetivas e ético-políticas. Psic teor e prátic. São Paulo, v. I7, n. I, p. 6I-71. 2015.

Silva TR, Carvalho EA. Depressão em professores universitários: uma revisão da literatura brasileira. Rev Un. Vol.28, n.I, pp.II3-II7. 2016. 
Silva AS, Borges, RSS. Adoecimento laboral das docentes da Universidade Federal do Rio de Janeiro e a relação com a divisão sexual do trabalho. Seminário Internacional Fazendo Gênero. Women's Worlds Congress (Anais Eletrônicos). Florianópolis, ISSN 2179 - 510X. 2017.

Silvério $M R$ et al. $O$ ensino na área da saúde e sua repercussão na qualidade de vida docente. Rev Bras de Educ Méd, 34(I), 65-73. 2010.

Tardif M. Saberes docentes e formação profissional. Editora vozes. 8 ed. Petrópolis- RJ, 2007. P.50. 2007.

Tardif M, Lessard C. O trabalho docente hoje: elementos para um quadro de análise. Petrópolis-RJ: Editora Vozes, $9^{\circ}$ Ed, p.12-13. 2007.

Viana AG, Barreto IG, Nunes NA.2013. Perfil de afastamento, por motivos de saúde, de servidores públicos federais da universidade federal do recôncavo da Bahia, de acordo com dados do SIASS. Rev cogn, n.I. 2013.

Vilela EF. 2010. Vivências de prazer-sofrimento no trabalho docente: um estudo em uma IES pública de Belo Horizonte. Mestrado em Administração. Faculdade Novos Horizontes, Belo Horizonte (MG) Brasil. 2010. 